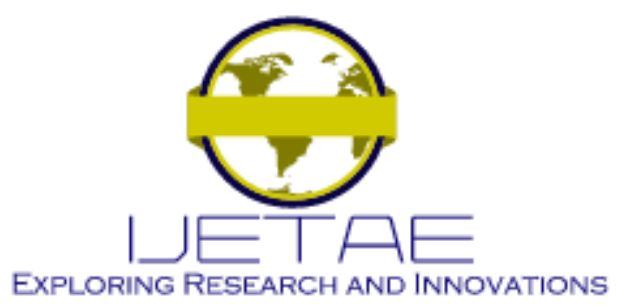

International Journal of Emerging Technology and Advanced Engineering

Website: www.ijetae.com (E-ISSN 2250-2459, Scopus Indexed, ISO 9001:2008 Certified Journal, Volume 11, Issue 11, November 2021)

Manuscript Received: 15 October 2021, Received in Revised form: 06 November 2021, Accepted: 10 November 2021

DOI: 10.46338/ijetae1121_03

\title{
Collision Avoidance for a Car-like Mobile Robots using Deep Reinforcement Learning
}

\author{
Kiwon Yeom \\ Department of Human Intelligence and Robot Engineering, Sangmyung University, South Korea
}

\begin{abstract}
The applications of mobile robots are more and more diverse and extensive. The motion planning of the mobile robots should be considered in aspect of effectiveness of the navigation, and collision-free motion is essential for mobile robots. In addition, dynamic path planning of unknown environment has always been a challenge for mobile robots. Aiming at navigation problems, this paper proposes a Deep Reinforcement Learning (DRL) based path planning algorithm which can navigate nonholonomic car-like mobile robots in an unknown dynamic environment. The output of the learned network are the robot's translational and angular velocities for the next time step. The method combines path planning on a 2D grid with reinforcement learning and does not need any supervision. The experiments illustrate that our trained policy can be applied to solve complex navigation tasks. Furthermore, we compare the performance of our learned controller to the popular approaches.
\end{abstract}

Keywords - Deep reinforcement learning, path planning, , artificial neural network, mobile robot, autonomous vehicle

\section{INTRODUCTION}

Path planning is the essential part for mobile robot navigation and control [1]. The objective of path planning is to find an optimal path avoiding collision with minimum required time from the starting position to the goal position.

The generated path for mobile robots should be as smooth as possible to meet the dynamics of the mobile robot as well. Most path planning algorithms of mobile robots rely on the information of the surrounding environment.

In general, mobile robots cannot complete their learning about environment as well as judgment in complex or cluttered environments [2]. Therefore, it requires to develop a path planning method using sparse information of the surrounding environment that mobile robots can quickly adapt to the surrounding environment and autonomously drive to the destination with safety.
As an alternative machine learning method for solving above issue, Deep Neural Networks (DNN) has drawn attention to researchers and there are many applications related to mobile robot's path planning [3].

The DNN algorithm has shown good performance in path planning and requires less prior information about the environment. Unlike the other supervised learning methods such as regression and back propagation, reinforcement learning does not require much sample data for training process.

Recently, to improve the navigation performance of mobile robots, several fusion algorithms were devised [4]. Although the performance of these applications are not outstanding, these fusion algorithm has obvious advantages in mobile robot's path planning.

From this observation, this paper proposes a mobile robot path planning system based on Deep Reinforcement Learning (DRL) controller.

DRL is a machine learning algorithm that combines Deep Learning (DL) and Reinforcement Learning (RL). RL takes into account the problem of agents (software agent, autonomous robot itself, etc) learning how to achieve their goals or make decisions. That is, DRL unites deep learning into solution, allowing agents to decide what actions to perform for optimizing the objective such as maximize the object function or minimizing the cost function.

In this paper, the current state from neural networks is mapped into the action of the reinforcement learning, and is updated through the enhancement function. The combined algorithm is verified by comparing with other path planning algorithms.

\section{Kinematic Model Of A Car-Like Mobile Robot}

The path planning considered in this article is based on a car-like mobile robot [5]. In general, the path has different conditions such as straight line, circular arc, turning, etc. 


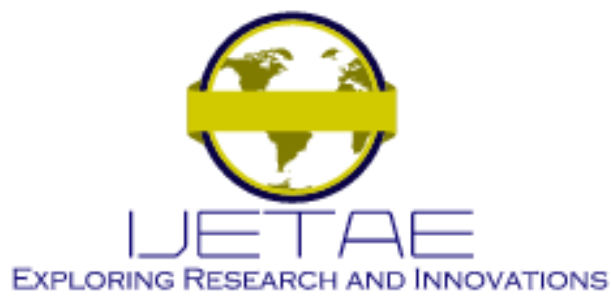

International Journal of Emerging Technology and Advanced Engineering

Website: www.ijetae.com (E-ISSN 2250-2459, Scopus Indexed, ISO 9001:2008 Certified Journal, Volume 11, Issue 11, November 2021)

The mobile robot can autonomously control the velocity, acceleration and steering angles to successfully achieve the given path tracking. For the purpose of controlling the mobile robot, in this paper a general kinematic bicycle model is applied to the car-like mobile robot. In this section, the brief explanation for the kinematic model is introduced, and more readings of kinematic bicycle model should be referred in [6].

A four-wheeled vehicle with a fixed rear wheels and front steering wheels can be approximated to the general bicycle model. Therefore, for the purpose of simplicity as in Figure 1. the control mechanism for the car-like mobile robot with the steering handle is analogous to that of the general bicycle.

The bicycle kinematic model, in this paper, is called the front wheel steering model because the front wheel orientation must be controlled to achieve the appropriate direction of the vehicle. On the contrary to this, the rear wheels are linked together through an axle, which is fixed to the vehicle and rotated with no motion of rotation such as roll, pitch and yaw. This study assumes that the wheel rolls without slipping sideways as well. For front-wheel steering of the vehicle, this paper supposes that the plane which the front wheels contact rotates with respect to the vertical axis from the ground as the handle is turned.

The pose of the mobile robot is described in the coordinate frame $B$ of which the origin is attached on the center of the rear axle with the forward direction according to the $x$-axis as shown in Figure 1 (left image).

In general, autonomous mobile robot's kinematic bicycle model can be represented by the continuous time nonlinear systems and it can be simply described as shown in Figure 1 (right image). More detail contents for the kinematic bicycle model can be referred in [6].
The mobile robot can be described by the generalized coordinates as follows

$$
q=(x, y, \theta) \in C
$$

Where $C \subset R^{2} \times S^{1}$. C represents the configuration space of the mobile robot and $S^{1}$ means unit circle with a set of angles $[0,2 \pi)$.

While the mobile robot can change the velocity of the wheels to generate rolling motion, the robot must be able to rotate about a reference point that is a common point perpendicularly lies along the wheel axis. The dashed lines describe the direction along which the wheels cannot move. As shown Figure 1, the lines of no motion are intersected at a point which is known as the ICR (Instantaneous Center of Rotation), and the mobile robot rotates about this point. Thus, the reference point, which is lies on the center of the mobile (see Figure 1. (b)), must follow a circular path as the mobile robot makes a turn. Consequentially, the angular velocity is generated represented as follows

$$
\theta=\frac{V}{\mathbb{R}_{B}}
$$

And by simple geometric the turning radius is

$$
R_{B}=L / \tan y
$$

Where $L$ is the length between the front and rear wheel bases. This simply means that the turning circle increases as the length is larger. 


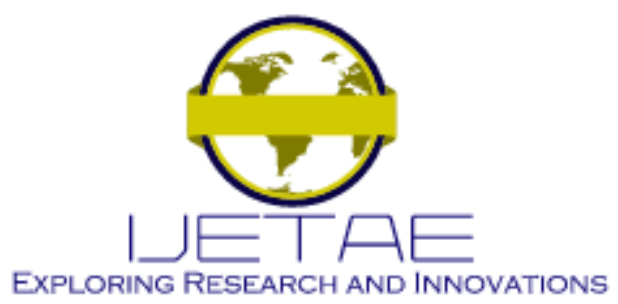

International Journal of Emerging Technology and Advanced Engineering

Website: www.ijetae.com (E-ISSN 2250-2459, Scopus Indexed, ISO 9001:2008 Certified Journal, Volume 11, Issue 11, November 2021)
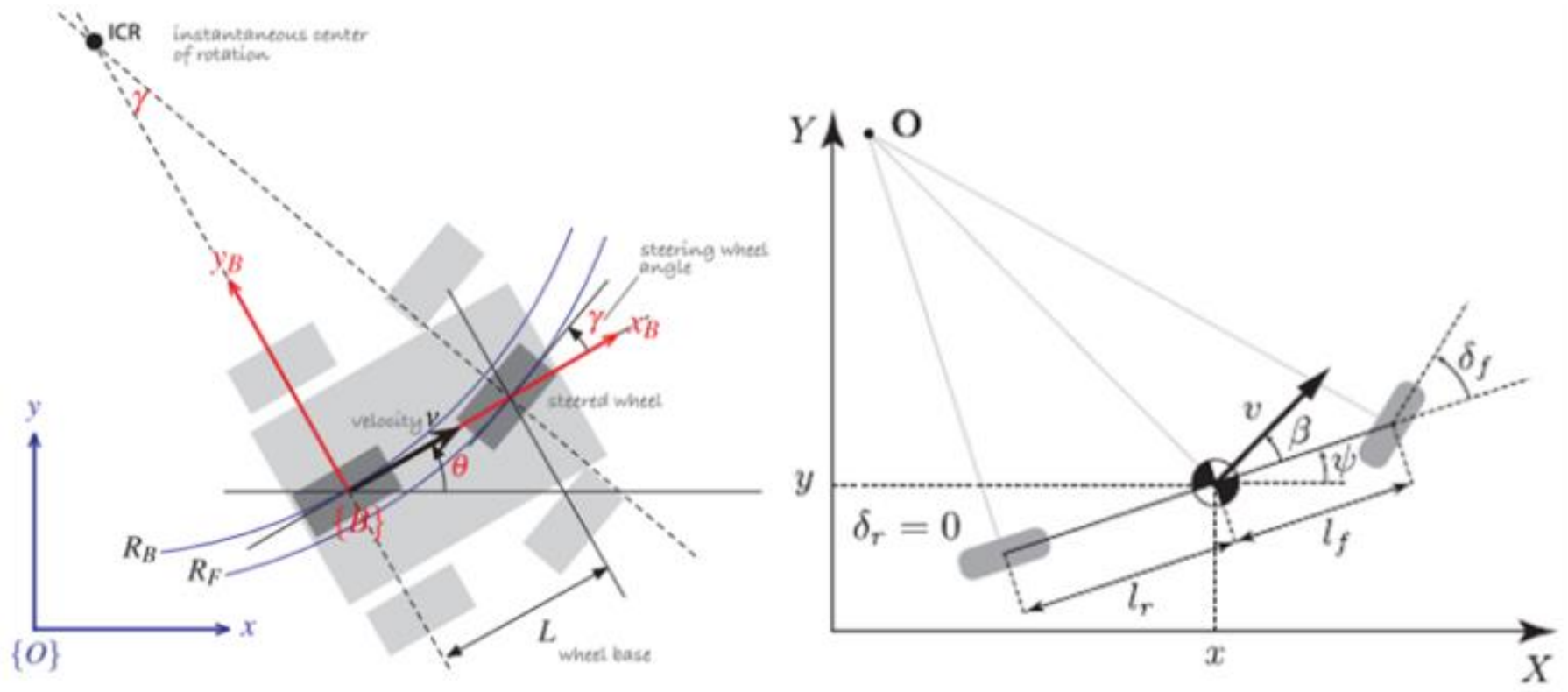

Figure 1. Bicycle model of a car-like mobile robot (figure courtesy of [3]).

The steering angle $\gamma$ is mechanically restricted, and the maximum value of the steering angle has the minimum value of $R_{B}$.

The lateral dynamics of bicycle model is as follows

$$
\begin{aligned}
\quad \dot{x}=v \cos (\psi+\beta) \\
\dot{y}=v \cos (\psi+\beta) \\
\dot{\psi}=\frac{v}{\mathbb{l}_{r}} \sin \beta \\
\dot{v}=\alpha \\
\beta=\tan ^{-1}\left(\frac{\mathbb{l}_{r}}{i_{f}+l_{r}} \tan \delta_{f}\right)
\end{aligned}
$$

where $x$ and $y$ are the coordinates of the center of mass of the mobile robot which are represented in Figure 1 (right).

$\psi$ is the heading angle of the mobile robot in an inertial frame and $v$ is the speed of the center of mass of the mobile robot. $l_{f}$ describes the distance from the center of the mass to the front, and and $l_{r}$ means the distance from the center of the mass to rear axles.

Compared to other models for autonomous mobile vehicles [7], it is easier to describe the vehicle system on the kinematic bicycle model since there are only two control parameters such as $l_{f}$ and $l_{r}$.
In addition, it is possible to design the path planner for the mobile robot with minor modification and build a similar controller for differently sized autonomous mobile vehicles.

$\beta$ is the angle from the center of mass with respect to the longitudinal axis of the mobile robot. It is also the same as the angle of the current velocity $v$ of the mobile robot. $\alpha$ is the acceleration of the center of mass of the mobile robot of which the direction is the same as the velocity.

In this lateral dynamics of bicycle model, the major control values are considered as the steering angles of the front wheels, namely, $\delta_{f}$ and $\alpha$. Since, in this paper, the mobile robot of which the rear wheels are fixed to the chassis of the vehicle, wherefore the rear wheels cannot be steered, namely $\delta_{r}=0$.

It should be considered in terms of the inertia for more accurate dynamics of the mobile robot. The position coordinates (Eq. 3 and 4) and heading angle (Eq. 5) with respect to the inertia of the dynamic bicycle model can be defined in the same manner as those in the velocity kinematic bicycle model (refer [8], for more specific descriptions).

The differential equations for the mobile robot are as follows

$$
\bar{x}=\not{\psi} y \alpha_{x}
$$




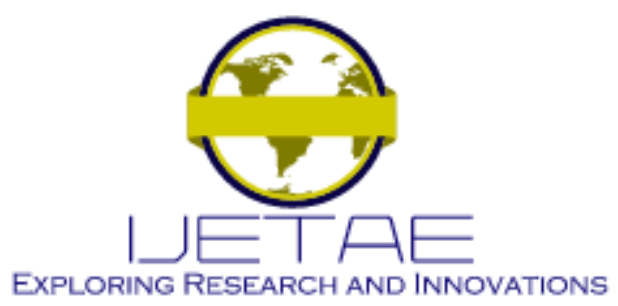

International Journal of Emerging Technology and Advanced Engineering Website: www.ijetae.com (E-ISSN 2250-2459, Scopus Indexed, ISO 9001:2008 Certified Journal, Volume 11, Issue 11, November 2021)

$$
\begin{aligned}
& \dot{y}=-\dot{\psi} \dot{x}+\frac{2}{m}\left(F_{c_{u} f} \cos \delta_{f}+F_{c y}\right) \\
& \dot{\psi}=\frac{2}{l_{z}}\left(l_{f} F_{c_{u} f}-l_{y} F_{c_{y}}\right) \\
& \dot{X}=\dot{x} \cos \psi-\dot{y} \sin \psi \\
& \dot{Y}=\dot{x} \sin \psi+\dot{y} \cos \psi
\end{aligned}
$$

where $\dot{x}$ denotes the longitudinal speed and $\dot{y}$ represents the lateral speed in the body frame of the mobile robot, and $\psi$ describes the ratio of the yaw rotation.

$F_{c, f}$ and $F_{c, r}$ describe the lateral forces of tires at the front and rear wheels, respectively, in the corresponding coordinate frame aligned with the plane of the paired wheels.

For the linear tire model, $F_{c, i}$ is defined as follows

$$
F_{c_{i} i}=-C_{\widetilde{C}_{i}} \alpha_{i}
$$

where $i \in\{f, r\}, \alpha_{\mathrm{i}}$ is the tire slip angle and $C_{\alpha \mathrm{i}}$ is the tire cornering stiffness.

\section{Design Of ARTIFICIAL NeURAL NETWORK}

In this paper, the deep reinforcement learning based navigation system consists of sensing data, 2D map, local path planner, and DRL controller which is comprised of DRL processor, which can generate the parameters such as velocity and steering angle, and vehicle controller which is used to control the car-like mobile robot as shown in Figure 2.

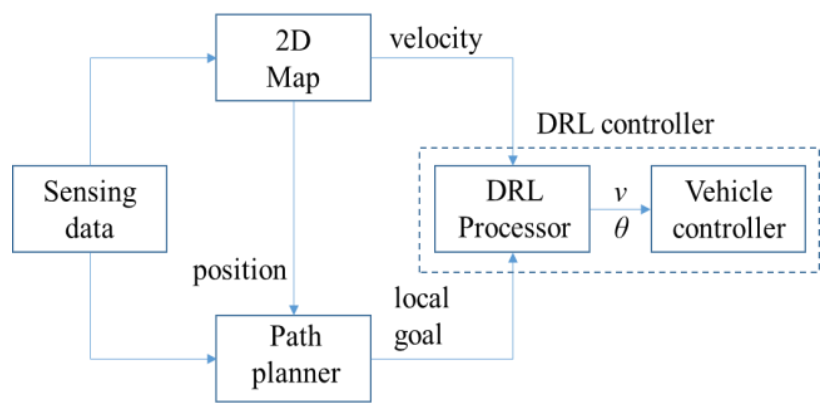

Figure 2. Block diagram of the DRL based mobile robot navigation.

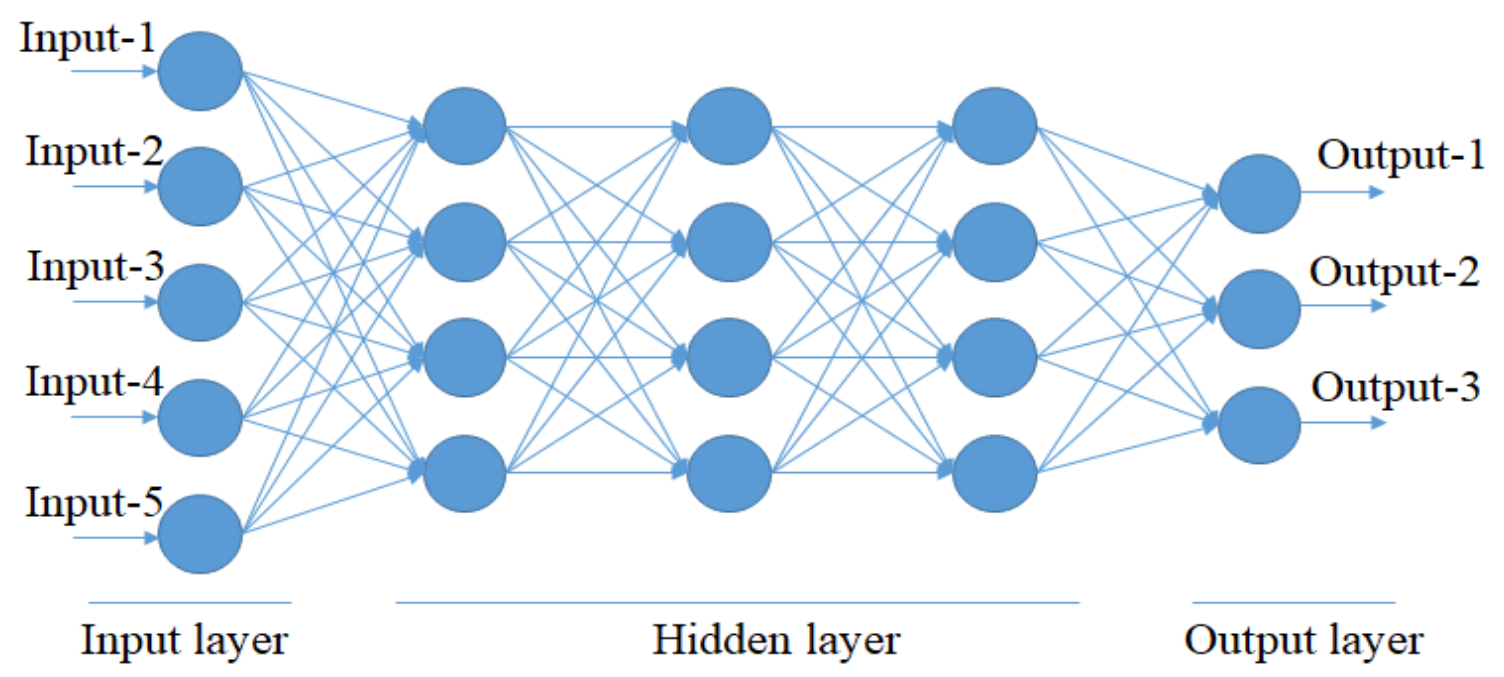

Figure 3. Demonstration of neural networks with four hidden layer

The path planner which generates the local goal position, and the vehicle controller which operates the mobile robot with the generated linear velocity and steering angle. The achievement of the driving speed is controlled by the velocity control module which depends on the bicycle kinematics model of the mobile robot.
ANN (Artificial Neural Networks) uses the traditional backpropagation and transformation scheme due to the remarkable ability of nonlinear mapping and fault tolerance. In addition, ANN comprises multiple layers with multiple neurons (see Figure 3) consisting of an input layer, a hidden layer, and an output layer as shown in Figure 3 [9]. 


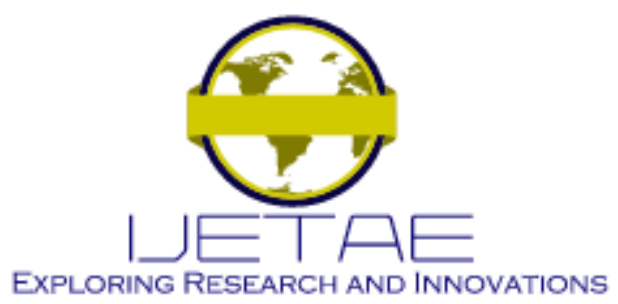

International Journal of Emerging Technology and Advanced Engineering

Website: www.ijetae.com (E-ISSN 2250-2459, Scopus Indexed, ISO 9001:2008 Certified Journal, Volume 11, Issue 11, November 2021)

The input vector is should be:

$$
x=\left[x_{1}, x_{2}, \ldots, x_{j, \ldots,} x_{m}\right] \quad j=1,2,3, \ldots m
$$

and the output vector should be:

$$
y=\left[y_{1}, y_{2}, \ldots, y_{k}, \ldots, y_{n}\right] \quad k=1,2,3, \ldots n
$$

The input neuron of the hidden layer is as follows

$$
\begin{gathered}
l_{j}^{[i]}=f\left(\text { net }_{j}^{[i]}\right) \\
\operatorname{net}_{j}^{[i]}=\sum_{i=1}^{j-1}\left(w_{i j}^{[i]} \cdot l_{j}^{[i-1]}+b_{j}^{[i]}\right) \\
i=1,2, \ldots, p, \quad j=1,2, \ldots, q
\end{gathered}
$$

where $i$ is the number of neurons in layer $i^{\text {th }}$ and $j$ is the number of layers, and $w_{i j}^{[\mathrm{in}]}$ is weights which connect the nodes of the $(j-1)^{\text {th }}$ layer to the nodes of $j^{\text {th }}$ layer, and $b$ is the bias value for nodes of the $j^{\text {th }}$ layer.

In this model, the hyperbolic tangent transfer function is considered which has the value in the range of -1 to +1 . The output layer uses linear transfer function (Purelin). For the learning, Traingdx package is used to update the weights and biases by gradient descent algorithm with adaptive learning rate. The performance is evaluated using mean squared error (MSE), where the number of epoch is set to 1000 times and the accuracy is set to 0.0001 .

In this paper, a deep neural network consists of a fully connected and multilayered that all the nodes, or neurons, in one layer are connected to the neurons in the next layer, and its diagram is drawn in Figure 3. Since fully connected networks is structure agnostic, in this paper, any special or additional assumptions not required for the input.

\section{Model OF REINFORCEMENT LEARNING}

In this section, the necessary part of the reinforcement learning is introduced for the architecture of the DRL based controller design. Further explanation of the reinforcement learning should be referred in [10].

An agent acquires information of the surrounding environments from a course of explorations. Then, the agent can execute an action to achieve its goal, and the agent will get evaluation, called feedback, of the action form the environment.
The evaluation can be considered as reward that the agent receives and as an indicator of the effect on the action which the agent performed. This process called 'trial and error' continuously tries to find the optimal action to achieve the maximum reward value [8]. This reward value also relates to the quality of the agent's action strategy. In other words, the larger the value is, the better the performed action is. Otherwise it will be degenerated or has a poor impact. This interaction process with the surrounding environment can be considered as a Markov Decision Processing (MDP) [11].

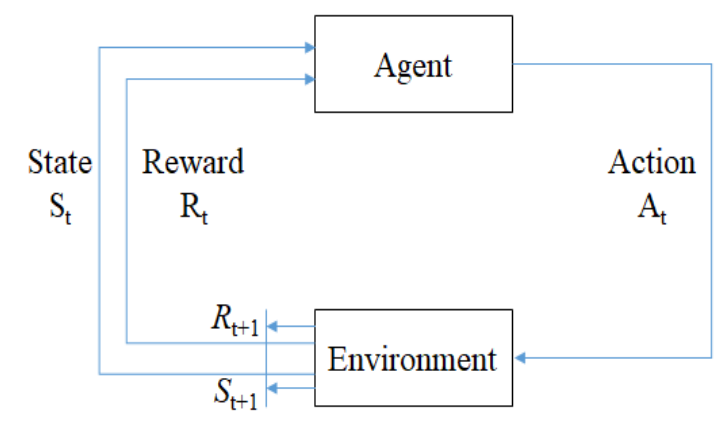

Figure 4. The schematic diagram of the reinforcement learning model.

At each timestamp $t$, system parameters are given by sensing data $s_{t}$, a local goal position $g_{t}$ at the $2 \mathrm{D}$ cartesian coordinate system, the linear velocity $v_{t}$ and angular velocity $\omega_{t}$ of the mobile robot. The policy which the mobile robot can select to avoid obstacles provides an action command at as follows

$$
\pi=a_{t} \in A\left(s_{t}\right), \quad s_{t} \in S
$$

where $S$ represents state set. The policy set is required to obtain the avoiding strategy $\pi$ "called the optimal policy.

If the set $\left\{S_{1}, S_{2}, S_{3, \ldots z} S_{t}\right\}$ satisfies the following equation, this set has Markov properties.

$$
\begin{gathered}
P_{F}\left(S_{t+1}=s^{t} \mid S_{t}=s_{t}, S_{t-1}=s_{t-1, \ldots,} S_{1}=s_{1}\right) \\
=P_{F}\left(S_{t+1}=s^{t} \mid S_{t}=s_{t}\right)
\end{gathered}
$$

In Markov process, if the state $s$ is decided, the actions are independent of each other. In other words, any actions which occurs in preceding state, are not correlated to the actions in posterior state. MDP is defined as the tuple $(S, O$, $A, T, R, \gamma)$ as follows 


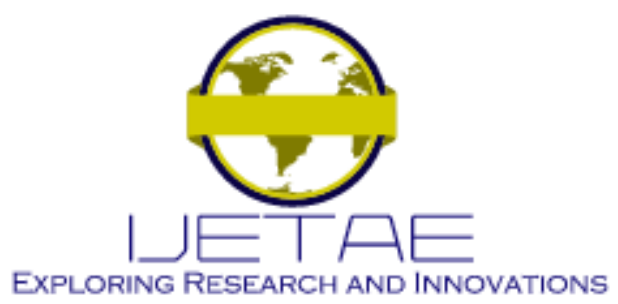

International Journal of Emerging Technology and Advanced Engineering

Website: www.ijetae.com (E-ISSN 2250-2459, Scopus Indexed, ISO 9001:2008 Certified Journal, Volume 11, Issue 11, November 2021)

$$
\begin{aligned}
& \left.\lambda\left(s^{s} \mid s_{v}, a, t, r_{s} \gamma\right)\right) \\
& =P_{F}\left(S_{t+1}=s^{s} \mid S_{t}=s_{s} \quad o_{t}=a_{x} A_{t}=a_{x}\right. \\
& \left.\quad T_{t}=t_{s} R_{t}=r s \gamma\right)
\end{aligned}
$$

where $S, A, R, T, O$ represent the state set, the action set, the reward, the transition, and the observation, respectively. The agent cannot determine the state $S$ but has to rely on observations $O\left(o \mid s^{b}, a\right)$. After each state transition, the robot receives an immediate reward $R(o \mid s, a)[12]$.

The state of the environment changes based on the robot's actions $a \in A$, which are in this paper the velocity, steering angle commands $(v, \theta)$, and the transition probability $T\left(s^{t} \mid s_{x} a\right)$. The output of sequential decisions can be regarded as a trajectory which the mobile robot has to drive from the start (previous) position $p_{0}$ to the goal (current) position $\mathrm{pg}_{g}$ with the traveling time $t_{g}$.

The objective of the control in this paper is to minimize the actual traveling time without any collision with obstacles and the formula is as follows

$$
\begin{aligned}
\arg \min _{\pi_{\theta}} \Psi\left[t_{g} \mid a_{t}\right. & =\pi_{\theta}\left(o_{t}\right) . \\
& \left.p_{t}=p_{t-1}+a_{t} \cdot \Delta t\right]
\end{aligned}
$$

where $\Theta$ is model parameters.

In general, the policy $\pi(a \mid s)$ of the mobile robot should define the probability which maps state $s$ to action $a$ [13]. A stochastic policy $\pi\left(a_{t} \mid o_{t}\right)$ can be evaluated by state value function $V^{\mathrm{\pi}}$ as follows

$$
V^{*}\left(s_{t}, a_{t}\right)=\max _{\pi} \Psi V^{\pi}(s, a)
$$

The reward function in this paper considers task completion $\left(R_{g}\right)$, the collision $\left(R_{e}\right)$, and the progress $\left(R_{p}\right)$ towards the goal position:

$$
R(s, a)=R_{g}(s, a)+R_{e}(s, a)+R_{p}(s, a)
$$

If the mobile robot reach its goal, it is awarded by $R_{g}(s, a)$ as follows

$$
R_{g}(s, a)=\left\{\begin{array}{rc}
\zeta & \text { if the goal was reached } \\
-\zeta & \text { if a collison accured } \\
0 & \text { otherwise }
\end{array}\right.
$$

$R_{g}(s, a)$ has a large positive value if the distance to the final goal is less than $0.5 \mathrm{~m}$. If the distance between the robot and the nearest obstacle is less than rotational radius, $R_{f i n}(s, a)$ has a large negative value since the collision can be occurred. $R_{e}(s, a)$ is a fixed negative reward for the mobile robot to finish an episode as fast as possible. The third reward component is used to speed up the training process

$$
R_{p}(s, a)=\eta \cdot D(s, a)
$$

where $D(s, a)$ is the cost function according to the distance between the mobile robot and subgoal at $p_{t}$ and $\eta$ is a scaling factor in the range of 0 to 1 .

\section{EXPERIMENTAL ENVIRONMENTS}

To examine the performance of the proposed DRL based path planning architecture, this paper constructed a 2D grid map and the simulation environment in Python with Tensorflow library.

The simulation program was developed using Python 3.7.3 under Intel 4-core i5 $7500 \mathrm{CPU} 3.80 \mathrm{GHz}, 32 \mathrm{G}$ memory, Windows 10 operating system.

As shown in Figure 5, the chassis of the mobile robot applied a car-like design with 4 wheels. Since the axle of the rear wheels is fixed to the body, the rear wheels can only rotate back and forth without pitch, roll and yaw.

The directional control of the front wheels is based on a simplified Ackerman control. In Ackerman steering scheme, the front wheels are enforced to keep the parallel alignment all the time, even though the axle turns on a pivot which is the vertical center position on the steering rod which is connected to a servo-motor for steering the front wheels through a rack and pinion gear system. The servo-motor is controlled by a microcontroller (Raspberry Pi 3 with Quad Core 1.2GHz) using a pulse-width modulation (PWM) signal and is capable of steering angles of $-45^{\circ} \leq \delta \leq 45^{\circ}$ about front wheels.

\section{RESULTS AND DISCUSSIONS}

To train the neural network and generate a policy to follow a global path without collisions, we sampled the start and goal positions randomly across the free space with short distance at the beginning stage and later increased the distance including several obstacles. 


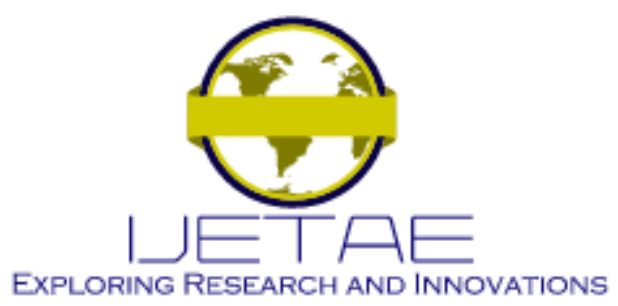

International Journal of Emerging Technology and Advanced Engineering

Website: www.ijetae.com (E-ISSN 2250-2459, Scopus Indexed, ISO 9001:2008 Certified Journal, Volume 11, Issue 11, November 2021)

After training, we performed experiments to evaluate the policy learned in terms of the success rate, which means that the mobile robot reached the goal position without any collisions and completion time. The performance was compared with Dynamic Window Approach (DWA) in ROS.

In the DWA, the control of the mobile robot is directly performed out through the velocity space which is a set of applicable velocities to the mobile robot under the surrounding environments. Therefore, to reduce the velocity space, the dynamics of the mobile robots is taken into account. In addition, the velocities, which are free with respect to the obstacles collision, are only considered as reachable candidates. Next, the velocity, which maximize the objective function or minimize the cost function of the control system, is chosen from the velocity space.

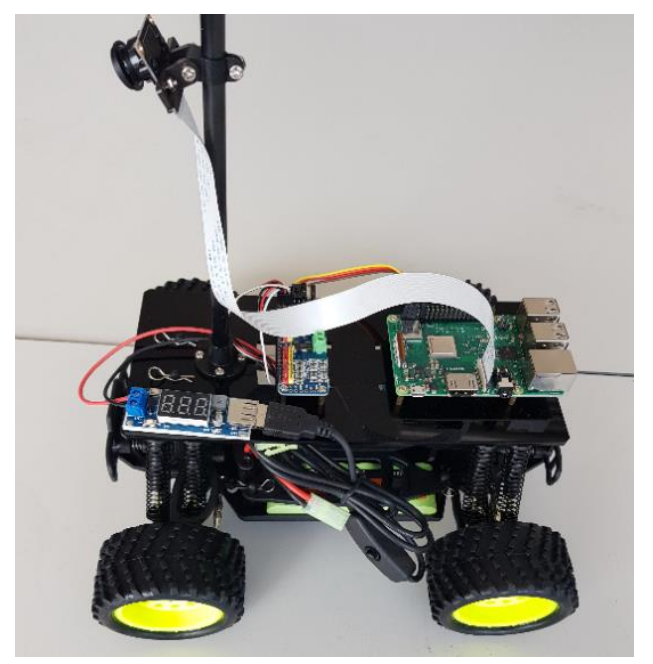

Figure 5. The autonomous car-like mobile robot platform.

A path from $(0,0)$ to $(200,200)$ is used to validate the proposed DRL algorithm. In this simulation, DWA is used for the purpose of the performance comparison [14].

As shown in Figure 6, both DWA and DRL algorithms reached the destination without any collisions.

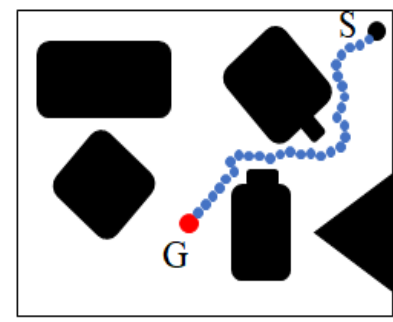

(a) DWA

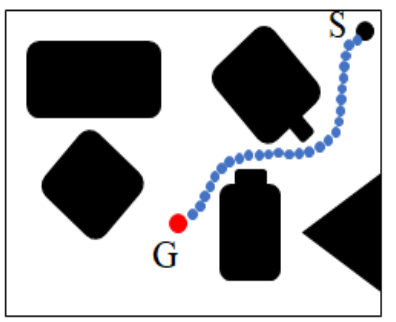

(b) The proposed
Figure 6. Experimental results of different path planning algorithms.

In addition, all methods effectively avoided obstacles under the different and more complex conditions as shown in Figure 7.

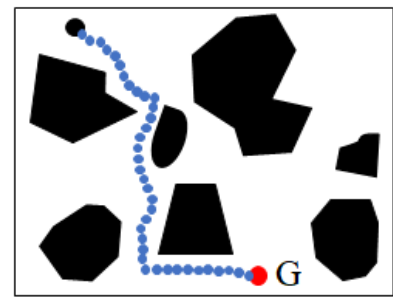

(a) DWA

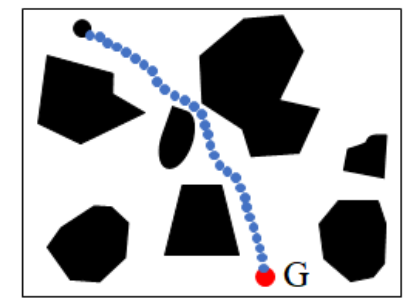

(b) The proposed
Figure 7. Path tracking in complex environments.

Comparing DWA, it was found that the proposed DRL based path planning efficiently reduced the number of path steps about 20\%. In addition, the proposed algorithm showed a less oscillation and rapid arrival time to the steady state as shown in Figure 8.

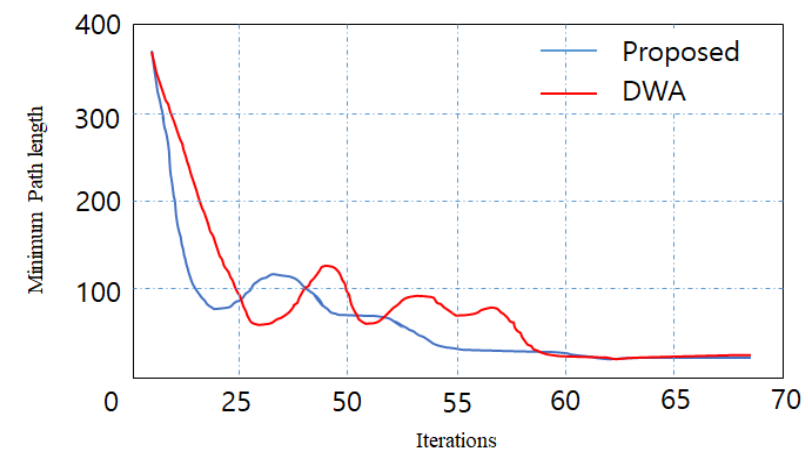

Figure 8. Path convergence curve. 


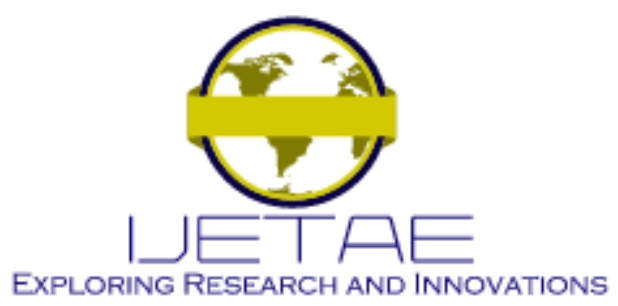

International Journal of Emerging Technology and Advanced Engineering

Website: www.ijetae.com (E-ISSN 2250-2459, Scopus Indexed, ISO 9001:2008 Certified Journal, Volume 11, Issue 11, November 2021)

As shown in Figure 8, the proposed algorithm has improvement over DWA in terms of success rate to reach the goal position.

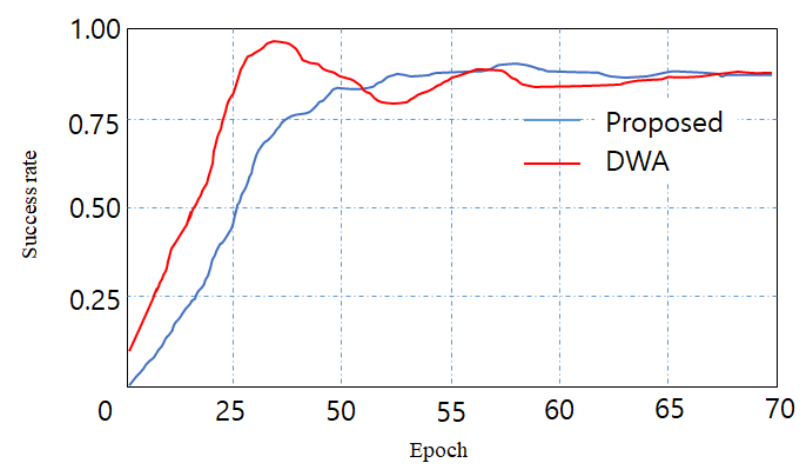

Figure 9. Success rate.

For each environment in Figure 9, 400 runs were performed with DWA and the proposed algorithm. in each testing environments. The success rates of DWA are lower than the proposed algorithm in all environments, especially in testing scenario 2 only reaching $60.4 \%$.

The path length of DWA has $20 \%$ longer than the proposed algorithm. However, the proposed algorithm has more time to complete navigation tasks, which shows the mobile robot controlled much cautiously about unknown environment as shown in Figure 10.

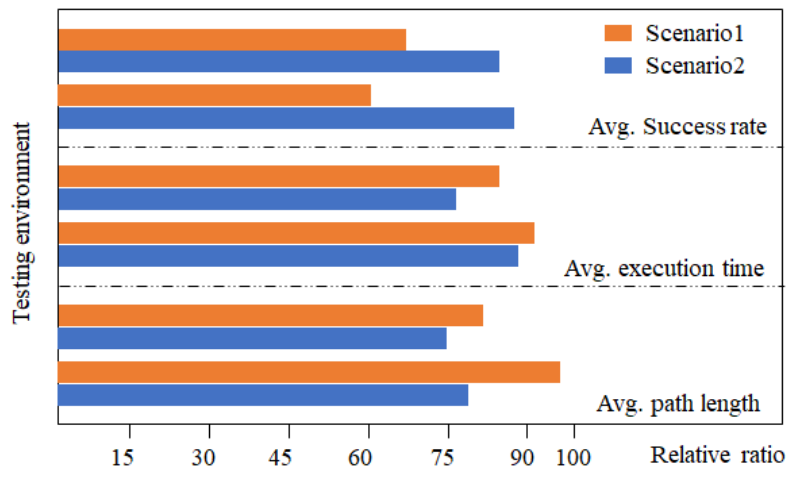

Figure 10. Performance evaluation with average of 400 runs.

\section{CONCLUSIONS}

This article introduced a deep reinforcement learning (DRL) based path planning architecture which was used for path following for the autonomous self-driving mobile robot.
This paper described the bicycle kinematic model for the mobile robot and DRL controller to track the given path.

The framework that combines the DRL processor and vehicle controller with the deep neural network was described. In order to verify the effectiveness of the proposed DRL based algorithm with collision avoidance, two different scenarios were considered.

In experimental results the proposed DRL controller showed better performance than DWA, and made more practical results. The DRL controller reduced the tracking errors and path length about $20 \%$ compared to the DWA controller in this article. For the future work, the study of effectiveness of the DRL controller should be performed in more complex environments with other control strategies.

\section{REFERENCES}

[1] N. H. Amer, H. Zamzuri, K. Hudha, and Z. Kadir. "Modelling and control strategies in path tracking control for autonomous ground vehicles: a review of state of the art and challenges", Journal of Advanced Robot and System, vol. 86, pp. 225-254, 2017.

[2] C. Shen, Y. Shi and B. Buckham, "Trajectory tracking control of an autonomous underwater vehicle using Lyapunov-based model predictive control," IEEE Transactions on Industrial Electronics, vol. 65, no 7, pp. 5796-5805, 2017.

[3] X. Yu, W. He, H. Li and J. Sun, "Adaptive Fuzzy Full-State and OutputFeedback Control for Uncertain Robots With Output Constraint", IEEE Transaction on Systems, Man, and Cybernetics, 2020, DOI: $10.1109 /$ TSMC.2019.2963072

[4] A. V. Duka, "Neural network based inverse kinematics solution for trajectory tracking of a robotic arm", Procedia Technol., vol. 12, no. 1, pp. 20-27, Jan. 2014.

[5] W. Gao, D. Hsu, W. S. Lee, S. Shen, and K. Subramanian. Intentionnet: Integrating planning and deep learning for goal-directed autonomous navigation. In CoRL, 2017.

[6] K.W, Yeom, "Kinematic and Dynamic Controller Design for Autonomous Driving of Car-like Mobile Robot", International Journal of Mechanical Engineering and Robotics Research, 2018. https://doi.org/10.1155/2018/3039061

[7] C. Urmson, J. Anhalt, D. Bagnell, C and Baker, R. Bittner, et al., Autonomous driving in urban environments: Boss and the urban challenge," Journal of Field Robotics, vol. 25, no. 8, pp. 425-466, 2008.

[8] N. H. Amer, H. Zamzuri, K. Hudha, and Z. Kadir, "Modelling and control strategies in path tracking control for autonomous ground vehicles: a review of state of the art and challenges." Journal of Intelligent \& Robotic Systems, vol. 86, no. 2, pp. 225-254, 2017.

[9] B. Karlik, A. V. Olgac, "Performance analysis of Various activation functions in generalized mlp architectures of neural networks, Int. J. Artif. Intell. Expert Syst., vol. 1, no. 4, pp. 111-122. 2011.

[10] S. Bansal, V. Tolani, S. Gupta, J. Malik, and C. Tomlin. Combining optimal control and learning for visual navigation in novel environments. In CoRL, 2019. 


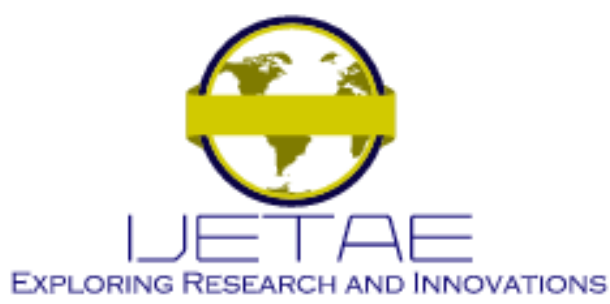

International Journal of Emerging Technology and Advanced Engineering

Website: www.ijetae.com (E-ISSN 2250-2459, Scopus Indexed, ISO 9001:2008 Certified Journal, Volume 11, Issue 11, November 2021)

[11] K. Chua, R. Calandra, R. McAllister, and S. Levine. Deep reinforcement learning in a handful of trials using probabilistic dynamics models. arXiv preprint arXiv:1805.12114, 2018.

[12] C. Berner, G. Brockman, B. Chan, V. Cheung, P. Debiak, C. Dennison, D. Farhi, Q. Fischer, S. Hashme, C. Hesse, et al. Dota 2 with large scale deep reinforcement learning. arXiv preprint arXiv:1912.06680, 2019.
[13] D. Kalashnikov, A. Irpan, P. Pastor, J. Ibarz, A. Herzog, E. Jang, D. Quillen, E. Holly, M. Kalakrishnan, V. Vanhoucke, et al. Qt-opt: Scalable deep reinforcement learning for vision based robotic manipulation. In CoRL, 2018.

[14] Özdemir A, Sezer V (2017) A hybrid obstacle avoidance method: follow the gap with dynamic window approach. In: 2017 First IEEE International Conference on Robotic Computing (IRC). 2017. 